\title{
A nearby young $M$ dwarf with a wide, possibly planetary-mass companion ${ }^{\star}$
}

\author{
N.R. Deacon $\dagger^{1}$, J.E. Schlieder ${ }^{2,3}$, S.J. Murphy ${ }^{4}$ \\ ${ }^{1}$ Centre for Astrophysics Research, University of Hertfordshire, College Lane, Hatfield, AL10 9AB, UK \\ ${ }^{2}$ NASA Postdoctoral Program Fellow, NASA Ames Research Center, Moffett Field, CA, USA \\ ${ }^{3}$ Max Planck Institute for Astronomy, Konigstuhl 17, Heidelberg, 69117, Germany \\ ${ }^{4}$ Research School of Astronomy and Astrophysics, Australian National University, Canberra, ACT 2611, Australia
}

\begin{abstract}
We present the identification of two previously known young objects in the solar neighbourhood as a likely very wide binary. TYC 9486-927-1, an active, rapidly rotating early-M dwarf, and 2MASS J21265040-8140293, a lowgravity L3 dwarf previously identified as candidate members of the $\sim 45 \mathrm{Myr}$ old Tucana Horologium association (TucHor). An updated proper motion measurement of the L3 secondary, and a detailed analysis of the pair's kinematics in the context of known nearby, young stars, reveals that they share common proper motion and are likely bound. New observations and analyses reveal the primary exhibits Li $6708 \AA$ absorption consistent with M dwarfs younger than TucHor but older than the $\sim 10 \mathrm{Myr}$ TW Hydra association yielding an age range of 10-45 Myr. A revised kinematic analysis suggests the space motions and positions of the pair are closer to, but not entirely in agreement with, the $\sim 24 \mathrm{Myr}$ old $\beta$ Pictoris moving group. This revised $10-45 \mathrm{Myr}$ age range yields a mass range of $11.6-15 \mathrm{M}_{J}$ for the secondary. It is thus likely 2MASS J2126-8140 is the widest orbit planetary mass object known ( $>4500 \mathrm{AU})$ and its estimated mass, age, spectral type, and $T_{\text {eff }}$ are similar to the well-studied planet $\beta$ Pictoris b. Because of their extreme separation and youth, this low-mass pair provide an interesting case study for very wide binary formation and evolution.
\end{abstract}

Key words: planets and satellites: detection, stars: binaries: visual, stars: brown dwarfs, stars: pre-main-sequence 


\section{INTRODUCTION}

Very wide orbit (>1000 AU) extrasolar planets represent a currently small but previously unexpected population of massive gas giant companions to stars. To date four such objects have been discovered by direct imaging by a variety of routes. WD 0806-661B (Luhman et al. 2011; $2500 \mathrm{AU} 6-9 \mathrm{M}_{J}$; Luhman et al. 2012) was discovered with a targeted observation of a nearby white dwarf, GU Psc b (Naud et al. 2014; $2000 \mathrm{AU}, 9-12 \mathrm{M}_{J}$ ) was found with a targeted observation of a young, nearby moving group member, SR12 C by observations of a T Tauri binary in $\rho$ Ophiuchus (Kuzuhara et al. 2011; 1100 AU, 6-20 M ${ }_{J}$ ), whilst Ross 458 C (Goldman et al. 2010; $1160 \mathrm{AU}, 5-14 \mathrm{M}_{J}$ ) was discovered in widefield survey data and then identified as having a common proper motion with its host binary.

In this work we present the identification of two previously known young objects in the solar neighbourhood, TYC 9486-927-1 and 2MASS J21265040-8140283, as a co-moving wide pair with a probable planetary mass secondary. During an examination of the literature we found that these two objects are separated by 217" and have similar proper motions. Hence we attempted to better determine their properties to see if they were a likely young, bound system. Using revised astrometry and detailed kinematic analyses of nearby young stars and brown dwarfs, we have determined that this previously known young brown dwarf/freefloating planetary mass object and young low mass star are a likely widely separated bound pair. We also present new spectroscopic observations and re-examinations of literature and archival data to refine the age of the system and estimate that the secondary is likely planetary mass and may be the widest orbit exoplanet yet discovered.

TYC 9486-927-1 was observed by Torres et al. (2006) as part of the Search for Associations Containing Young stars (SACY) programme (Torres et al. 2008). They assigned a spectral type of M1 and measured a radial velocity of $v_{\text {rad }}=8.7 \pm 4.6 \mathrm{~km} / \mathrm{s}$ from 10 observations. The large uncertainty is likely due to the star's high rotational velocity $(v \sin i=$ $43.5 \pm 1.2 \mathrm{~km} / \mathrm{s}$ ); suggesting it is either a single rapid rotator or a spectroscopic binary with blended lines. TYC 9486-927-1 also shows signs of activity in X-ray (Thomas et al. 1998), H $\alpha$ emission (Torres et al. 2006) and the UV (using GALEX data from Martin et al. 2005 we find $\left.\log F_{F U V} / F_{J}=-2.49, \log F_{N U V} / F_{J}=-2.11\right)$. 2MASS J2126-8140 is an L3 first identified by Reid et al. (2008) (although referencing Cruz et al. 2009 as the discovery paper).

\footnotetext{
* Based on observations made with the ESO/MPG 2.2m telescope at the La Silla Observatory under programme ID 090.A-9010.
} $\dagger$ E-mail:n.deacon2@herts.ac.uk 
Subsequently Faherty et al. (2013) classified it as a low gravity L3 $\gamma$ (using the gravity classification system of Cruz et al. 2009). Recent VLT/ISAAC observations by Maniavacas et al. (2014) find it is a good match to the young, L3 companion CD-35 2722B (Wahhaj et al. 2011). These authors also used the spectral indices of Allers \& Liu (2013) to confirm that the 2MASS J2126-8140 is an L3 and shows low gravity spectral features. Manjavacas et al. (2014) also used the BT-Settl-2013 atmospheric models (Allard et al. 2012) to derive $T_{\text {eff }}=$ $1800 \pm 100 \mathrm{~K}, \log g=4.0 \pm 0.5 \mathrm{dex}$, albeit with better fits to super-solar metallicity models. Filippazzo et al. (2015) use photometry, a trigonometric parallax of $31.3 \pm 2.6$ mas (referenced to Faherty et al., in prep.) and evolutionary models to derive an effective temperature of $1663 \pm 35 \mathrm{~K}$. They also derived a mass of $23.80 \pm 15.19 \mathrm{M}_{J}$ assuming a broad young age range of 10-150 Myr. Gagné et al. (2014) listed 2MASS J2126-8140 as a high probability candidate member of Tucana-Horologium association (TucHor) but noted that its photometric distance would be in better agreement with its TucHor kinematic distance if it were an equal mass binary.

\section{TYC 9486-927-1 AND 2MASS J21265040-8140293}

Our parameters for both components of this system are listed in Table 4. Below we outline how these were derived.

\section{$2.1 \quad$ Astrometry of 2MASS J21265040-8140293}

Using 2MASS and WISE astrometry, Gagné et al. (2014) measured proper motions of $\mu_{\alpha} \cos \delta=$ $46.7 \pm 1.3 \mathrm{mas} / \mathrm{yr}$ and $\mu_{\delta}=-107.8 \pm 10.4 \mathrm{mas} / \mathrm{yr}$ for $2 \mathrm{MASS}$ J2126-8140. This is deviant by $6 \sigma$ in the R.A direction from the UCAC4 measurements (Zacharias et al. 2013) for TYC 9486-927-1 of $\mu_{\alpha} \cos \delta=58.9 \pm 1.5 \mathrm{mas} / \mathrm{yr}$ and $\mu_{\delta}=-109.4 \pm 1.0 \mathrm{mas} / \mathrm{yr}$. Due to the very small uncertainty on Gagné et al. (2014)'s $\mu_{\alpha} \cos \delta$ measurement and the availability of newer datasets we recalculated the astrometric solution for 2MASS J2126-8140 using data from 2MASS (Skrutskie et al.|2006), the WISE All-Sky release(Wright et al.|2010), one epoch of WISE post-cryo data, one epoch of the reactivated NEO-WISE mission (Mainzer et al. 2011) and the DENIS survey (Epchtein et al. 1994). For each of the three WISE epochs we averaged the single exposure positional measurements to produce three datapoints. We assumed positional errors ( 84 mas on both axes) from the quoted errors on the position for 2MASS J2126-8140in the WISE All-Sky data release Source Catalogue and applied these to 

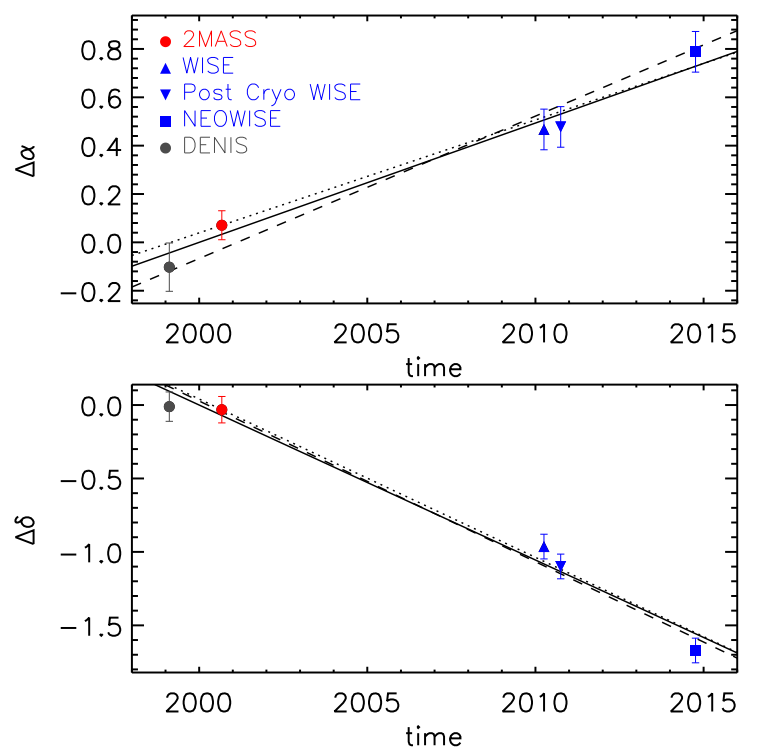

Figure 1. Our proper motion fit for 2MASS J2126-8140 using data from various infrared surveys. The solid line is our proper motion fit, the dashed line is the proper motion for TYC 9486-927-1 (Zacharias et al. 2013) shifted so it matches our proper motion at the midpoint of our dataset and the dotted line is the Gagné et al. (2014) proper motion extrapolated from the 2MASS position.

all three of our WISE datapoints 1. For 2MASS we used the quoted positions and positional error and for DENIS we used the approach of Luhman (2013), measuring the positional scatter on objects of similar brightness close to the target. This latter calculation yielded positional uncertainties of 100 mas in both R.A. and Dec. which were applied to positions averaged from the different DENIS epochs. These measurements were combined in a least squares fit which resulted in proper motion measurements of $\mu_{\alpha} \cos \delta=49.3 \pm 9.7 \mathrm{mas} / \mathrm{yr}$ and $\mu_{\delta}=-105.5 \pm 6.6 \mathrm{mas} / \mathrm{yr}$. These figures deviate by less than $1 \sigma$ from the UCAC4 proper motion measurements for TYC 9486-927-1 from Zacharias et al. (2013). Our proper motion fit along with those from Gagné et al. (2014) and the UCAC4 proper motion for TYC 9486-927-1 are shown in Figure1. The congruent proper motions are readily apparent on the plane of the sky in Figure 2 ,

\subsection{The radial velocity of 2MASS J21265040-8140293}

2MASS J2126-8140 was observed with the Phoenix spectrograph (Hinkle et al. 2003) mounted to the Gemini-South telescope on UT 2009 October 29 (Programme GS-2009B-C-2, PI K. Cruz). The observations consisted of two AB pairs with each exposure lasting 1800s. The data was obtained in the $\mathrm{H}$-band with the 0.34 arcsecond slit which provides a resolving

1 The WISE All-Sky Source Catalogue position is the average of multiple measurements at one of our epochs and thus the error on this averaged position will be representative of the error on our averaged position at each of our three WISE epochs. 


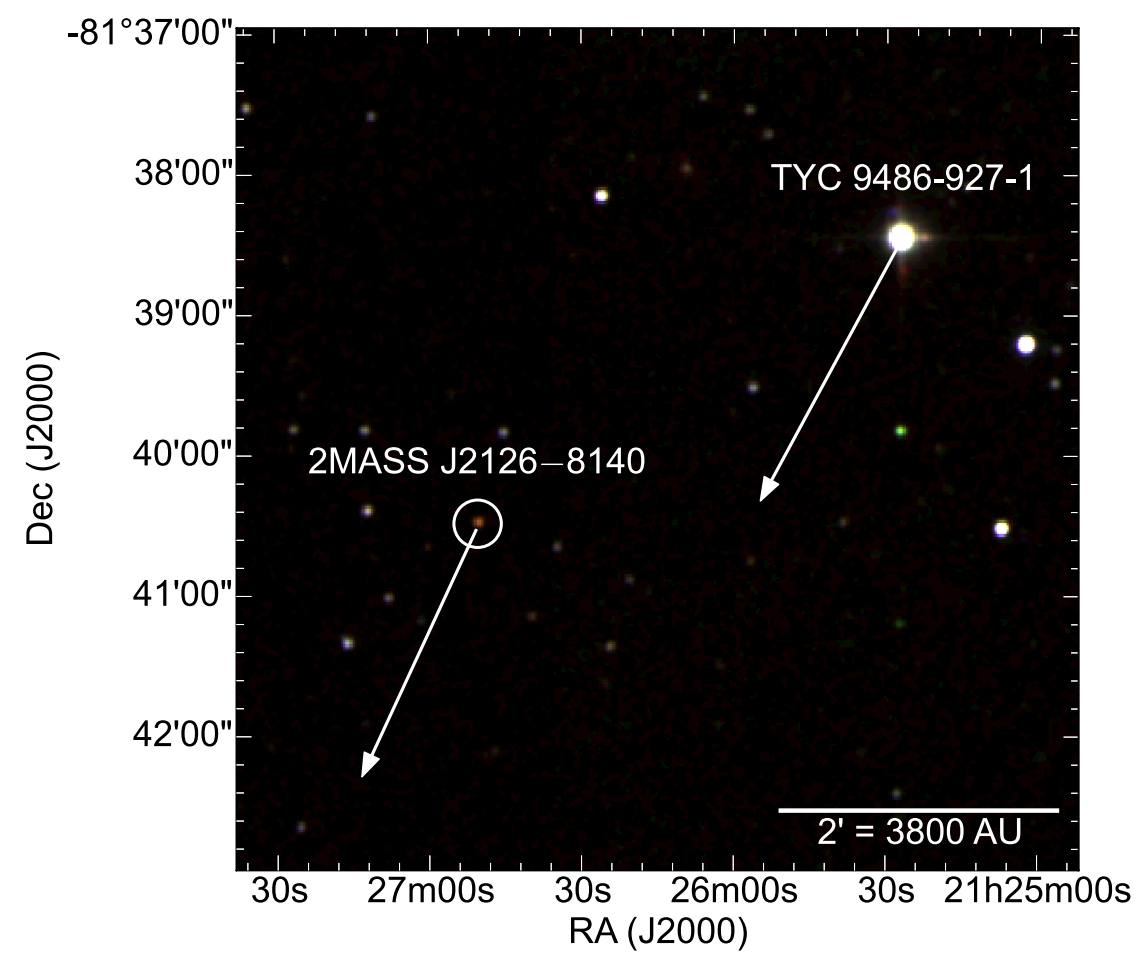

Figure 2. A $6 \times 6$ arcminute $2 M A S S J H K_{s}$ colour image of TYC 9486-927-1 and 2MASS J2126-8140 showing the direction of the proper motion vectors from Zacharias et al. (2013) and this work respectively. The length of the arrows shows the motion on the sky over 1000 years. The AU scale assumes the Filippazzo et al. (2015) distance of 31.9 pc.

Table 1. The radial velocity measurements derived from the low signal to noise Gemini/Phoenix spectrum of 2MASS J2126-8140 by cross-correlating with different spectral templates. The three spectral template stars come from Prato et al. (2002) and the model template from (Allard et al. 2011).

\begin{tabular}{lrc}
\hline Template & RV $(\mathrm{km} / \mathrm{s})$ & Cross-correlation power \\
\hline 1800 K model & $10.5 \pm 1.1$ & $18 \%$ \\
LHS 2065 (M9) & $7.4 \pm 1.8$ & $23 \%$ \\
LHS 2351 (M7) & $8.4 \pm 1.4$ & $24 \%$ \\
LHS 292 (M6) & $8.1 \pm 1.4$ & $23 \%$ \\
GL 644C (M7) & $7.6 \pm 1.5$ & $24 \%$ \\
\hline Adopted value & $8.4 \pm 2.1$ & \\
\hline
\end{tabular}

power of approximately 50,000. Along with the science data, flat lamp and dark calibration exposures were obtained on the same night. These data were downloaded from the Gemini Archive and reduced using a series of custom IDL routines. We corrected for bad pixels then flat-fielded and dark subtracted the science frames using a median master flat and dark frame. We attempted to extract the one dimensional spectrum from both sky-subtracted AB pairs, but the trace was only detected by our software in one pair. OH night sky lines were used to solve the dispersion solution and establish a wavelength scale. The final extracted spectrum covered 1.5512 - 1.5577 microns and had a SNR 5. Prior to cross correlation 
with model and observed templates, the spectrum was flattened by dividing by a 4 th order polynomial fit to the continuum to the continuum, flux normalized, and corrected for the barycentric velocity.

To measure the RV of $2 \mathrm{MJ} 2126$, we cross-correlated the spectrum with a $1800 \mathrm{~K}, \log (\mathrm{g})=4.0$, solar metallicity BT Settl (Allard et al. 2011) model spectrum (to match the parameters from Manjavacas et al. 2014) and observed M6, M7, and M9 Keck/NIRSPEC template spectra from Prato et al. (2002). Our analyses provide consistent RVs using each template, all four measurements are listed in Table 1. All measurements are in reasonable agreement with TYC 9486-927-1's RV $(10.0 \pm 1.0 \mathrm{~km} / \mathrm{s}$, see Section 2.3). We adopt a range of $8.4 \pm 2.1 \mathrm{~km} / \mathrm{s}$ for 2MASS J2126-8140's radial velocity based on our measurements. We note that this radial velocity estimate comes from a very low signal to noise spectrum. However we include this measurement to demonstrate that we have analysed the available archive data for 2MASS J2126-8140 and can find no data which suggests that it is not in a bound system with TYC 9486-927-1.

\subsection{Characterisation of TYC 9486-927-1}

TYC 9486-927-1 was classified as an active M1 by Torres et al. (2006) who also detected a Lithium $6708 \AA$ feature with an equivalent width of $104 \mathrm{~m} \AA$. We observed TYC 9486927-1 with FEROS (Kaufer et al. 1999; R=48,000, 3600-9200A) on 2012 October 7 using all the standard settings, reductions, and RV analyses detailed in sections 2.3 .7 and 3.11 of Bowler et al. (2015). We subsequently observed the star on 2015 August 26 and 2015 October 27 with the WiFeS instrument on the ANU $2.3 \mathrm{~m}$ telescope at Siding Spring using the R7000 grating (Dopita et al. 2007; $\mathrm{R}=7000,5250-7000 \AA$ ). The WiFeS instrument set up, data reduction and analysis, including the derivation of line widths and radial velocities, was the same as that described in Murphy \& Lawson (2014). Our FEROS spectrum showed emission in $\mathrm{H} \alpha, \mathrm{H} \beta, \mathrm{H} \gamma$ and $\mathrm{H} \delta$ and yielded measurements of $v_{\text {rad }}=10.0 \pm 1.0 \mathrm{~km} / \mathrm{s}, E W_{L i}=$ $85 \pm 15 \mathrm{~m} \AA$ and $v \sin i=40.0 \pm 2.0 \mathrm{~km} / \mathrm{s}$, while the WiFeS observations measured $v_{\text {rad }}=$ $10.7 \pm 1.0 \mathrm{~km} / \mathrm{s}$ and $9.7 \pm 1.0 \mathrm{~km} / \mathrm{s}$ respectively with $E W_{L i}=90 \pm 10 \mathrm{mÅin}$ both observations. Table2 shows the spectroscopic properties of TYC 9486-927-1 from our observations and the literature. Notably, the star exhibits no RV variations outside of uncertainties on the scale of months to years, strongly suggesting it is not a close to equal mass spectroscopic binary system. We adopt a radial velocity of $10.0 \pm 1.0 \mathrm{~km} / \mathrm{s}$ for the star, in agreement with the 
FEROS and WiFeS data, and lower precision measurements of $8.7+/-4.6 \mathrm{~km} / \mathrm{s}$ (Torres et al. 2006) and $11.9 \pm 3.0 \mathrm{~km} / \mathrm{s}$ (Malo et al. 2014). To further examine the possible binary nature of TYC 9486-927-1, we performed 2D cross correlations on our FEROS spectrum with a variety of primary/secondary template mass ratio combinations. None of these tests yielded a reliable cross-correlation function with power larger than in the case of a single star.

To garner an improved spectral type, we made an additional observation of TYC 9486927-1 with WiFeS on the 2015 November 28 with the lower resolution R3000 grating $(\mathrm{R}=3000$, 5200-9800A). We visually compared the flux calibrated and telluric corrected spectrum to spectral type standards from the lists of E. Mamajek 2 observed that night with the same instrument settings. Figure 3 shows that TYC 9486-927-1 has a spectral type between M2 (GJ 382) and M2.5 (GJ 381), inconsistent with the M1 spectral type reported by Torres et al. (2006). To compliment the visual comparison we also measured several molecular spectral type indices recently calibrated by Lépine et al. (2013). These are listed in Table 3 compared to measurements made from a low-resolution spectrum of TYC 9486-927-1 by Gaidos et al. (2014), who assigned a spectral type of M3 from visual inspection. Based on all available spectroscopic observations we assign a spectral type of M2.0 \pm 0.5 for TYC 9486-927-1. This also agrees with photometric spectral types obtained from $V-J$ (M2.3, Lépine et al. 2013) and $V-K_{s}$ (M1.8, Pecaut \& Mamajek 2013) colours.

Elliott et al. (2015) imaged TYC 9486-927-1 with the VLT/NACO AO imager and found no companion, despite being able to detect an equal mass companion down to a projected separation of $3 \mathrm{AU}$ (at an assumed photometric distance of $36.3 \mathrm{pc}$ distance or $4.2 \mathrm{AU}$ adjusting that photometric distance for equal-mass binarity). These observations and our multiepoch radial velocity data suggest that TYC 9486 is a single, rapidly rotating star rather than a spectroscopic or tight, visual binary. However, it is still possible that TYC 9486-927-1 is an equal mass binary with a face-on orbit and close separation.

\subsubsection{The age of TYC 9486-927-1}

TYC 9486-927-1 has rapid rotation and coronal and chromospheric activity suggestive of a young age. We measured a weak lithium $6708 \AA$ absorption feature in both our FEROS $(85 \pm 15 \mathrm{~m} \AA)$ and WiFeS spectra $(90 \pm 10 \mathrm{~m} \AA$, see Figure 3 inset) consistent with the $104 \mathrm{~m} \AA$ measurement of Torres et al. (2006). The detection of lithium is an important age diagnostic for early 
Table 2. Properties derived from multi-epoch spectroscopy for TYC 9486-927-1 from both the literature and our work. Note the consistency of the radial velocity over long periods and the variability in the $\mathrm{H} \alpha \mathrm{EW}$ due to chromospheric activity.. The last line refers to our lower resolution R3000 observation where the Lithium $6708 \AA$ feature was not resolved.

\begin{tabular}{lllcccc}
\hline Source & Date $(\mathrm{UT})$ & $\mathrm{SpT}$ & $\mathrm{RV}(\mathrm{km} / \mathrm{s})$ & $\mathrm{EW} \mathrm{Li}(\mathrm{m} \AA)$ & $\mathrm{EW} \mathrm{H} \alpha(\AA)$ & $v \sin i(\mathrm{~km} / \mathrm{s})$ \\
\hline Torres et al. (2006) & $2001-09-08$ & $\mathrm{M} 1 \mathrm{e}$ & $8.7 \pm 4.6$ & 104 & -5.6 & $43.5 \pm 1.2$ \\
Malo et al. (2014) & $2010-05-25$ & $\ldots$ & $11.9 \pm 3.0$ & $\ldots$ & $\ldots$ & $44.8 \pm 4.2$ \\
Gaidos et al. (2014) & $\ldots$ & $\mathrm{M} 3$ & $\ldots$ & $\ldots$ & -3.9 & $\ldots$ \\
This work, FEROS & $2012-10-07$ & $\ldots$ & $10.0 \pm 1.0$ & $85 \pm 15$ & $\ldots$ & $40.0 \pm 2.0$ \\
This work, WiFeS & $2015-08-26$ & $\ldots$ & $10.7 \pm 1.0$ & $90 \pm 10$ & $-5.7 \pm 0.2$ & $\ldots$ \\
& $2015-10-27$ & $\ldots$ & $9.7 \pm 1.0$ & $90 \pm 10$ & $-9.5 \pm 0.2$ & $\ldots$ \\
& $2015-11-28$ & $\mathrm{M} 2 \pm 0.5$ & $\ldots$ & $\ldots$ & $-6.0 \pm 0.5$ & $\ldots$ \\
\hline
\end{tabular}

Table 3. Spectral indices in the Lépine et al. (2013) format and the estimated spectral type from each value (in parenthesis). Our visual comparison is relative to the spectral standards of Mamajek 4 .

\begin{tabular}{|c|c|c|c|c|c|c|c|c|}
\hline Source & $\mathrm{CaH} 2$ & $\mathrm{CaH} 3$ & $\mathrm{TiO} 5$ & VO1 & $\mathrm{VO} 2$ & ColorM & Visual & Final \\
\hline Gaidos et al. (2014) & $0.613(\mathrm{M} 1.2)$ & $0.793(\mathrm{M} 1.6)$ & 0.697 (M1.2) & & & & M3 & \\
\hline This work & $0.574(\mathrm{M} 1.7)$ & $0.769(\mathrm{M} 2.1)$ & $0.591(\mathrm{M} 2.2)$ & $0.940(\mathrm{M} 2.7)$ & $0.886(\mathrm{M} 2.4)$ & $1.575(\mathrm{M} 3.1)$ & M2 & M2 \\
\hline
\end{tabular}

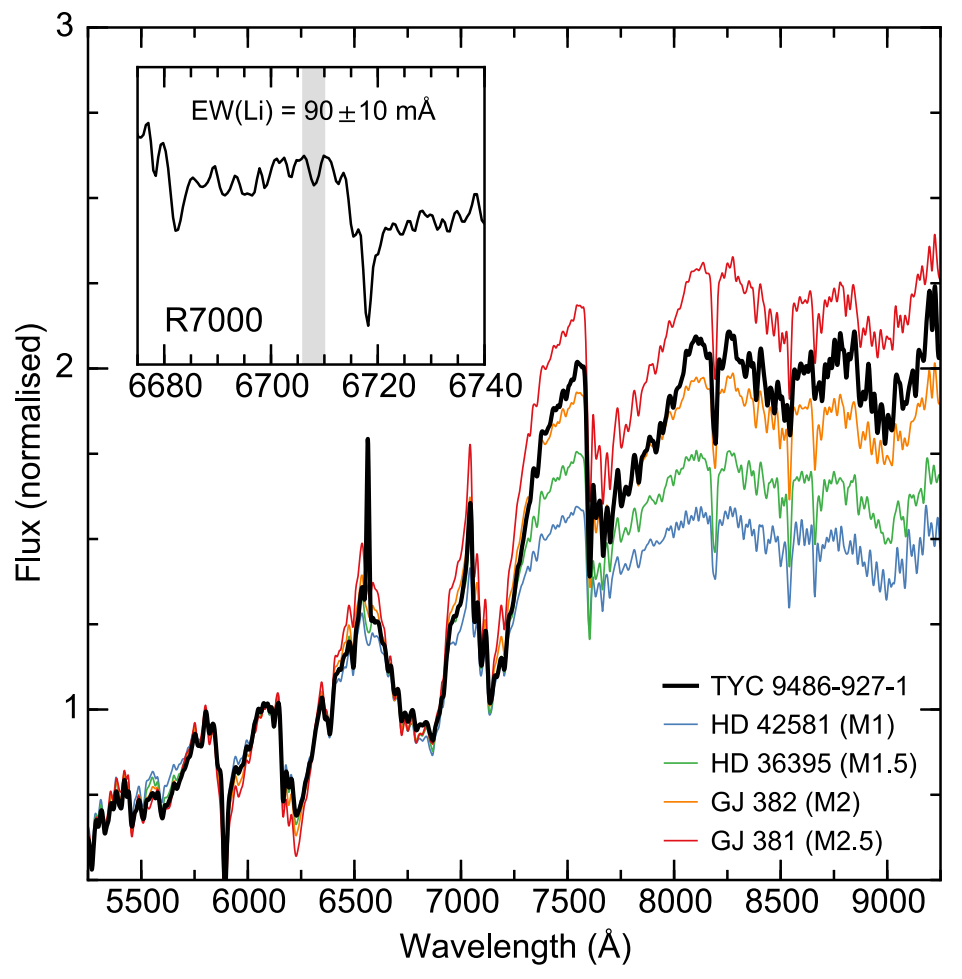

Figure 3. .WiFeS/R3000 spectrum of TYC 9486-927-1 (thick line), compared to spectral type standards observed the same night. All spectra have been normalised at $6100 \mathrm{~A}$ and smoothed with a 5-px Gaussian prior to plotting. Based on this comparison the spectral type of TYC 9486-927-1 is M2-M2.5. The inset shows the weak (EW=90 $\pm 10 \mathrm{~m} \AA$ ) and broad Li I $6708 \AA$ line from a higher resolution WiFeS R7000 observation. 


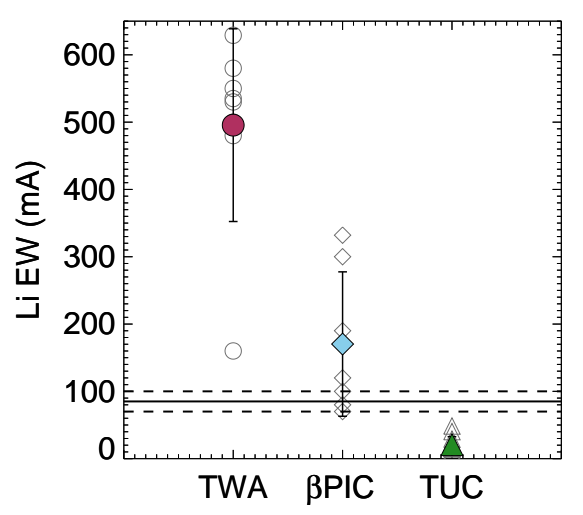

Figure 4. The lithium $6708 \AA$ absorption for TYC 9486-927-1(solid line with dashed line error bars) plotted against M1 and M2 members (da Silva et al. 2009) of the TWA (10 $\pm 3 \mathrm{Myr}), \beta$ Pic $(24 \pm 3 \mathrm{Myr})$ and TucHor (45 \pm 4 Myr) associations (all ages from Bell et al. 2015) with additional TucHor members from Kraus et al. (2014). The solid data points with error bars show the mean and standard deviation of each association. Clearly TYC 9486-927-1has a lithium absorption strength between members of TWA and TucHor and in agreement with $\beta$ Pic.

M-dwarfs, as the element is typically depleted in the photospheres of such stars on time scales of ¡40 Myr (e.g. Mentuch et al. 2008). To illustrate this, in Figure 4 we show the

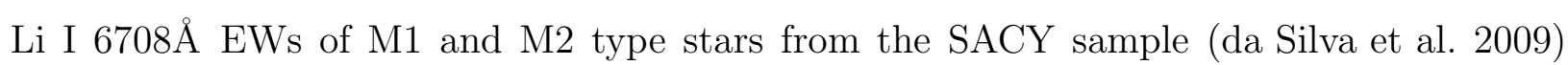

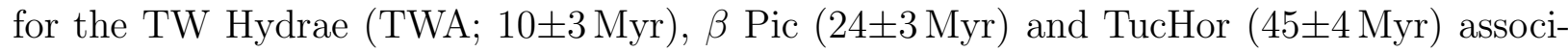
ations with additional TucHor members from Kraus et al. (2014) (all ages from Bell et al. 2015) against the EW for TYC 9486-927-1 . It is clear that the TYC 9486-927-1 has stronger lithium absorption than stars of similar spectral type in TucHor, weaker absorption than TWA members but in reasonable agreement with $\beta$ Pic members. Based on this comparison we conclude that TYC 9486-927-1 is older than TWA and likely of similar age or younger than TucHor. Thus, our Li analysis suggests an age comparable to the $\beta$ Pic moving group, but we note that Li depletion in low-mass stars can be affected by initial conditions (rotation, episodic accretion) and we therefore adopt a conservative age range of 10-45 Myr.

\subsection{Photometric distances and moving group membership}

TYC 9486-927-1 lacks a trigonometric parallax measurement and thus any determination of its kinematics (and hence moving group membership) requires photometric distance estimates. To estimate absolute near-IR magnitudes for TYC 9486-927-1 we used our measured spectral type of M2. We then derived an effective temperature of $3490 \mathrm{~K}$ for TYC 9486927-1 using the 5-30 Myr young star $T_{\text {eff }}$ scale of Pecaut \& Mamajek (2013) and applied this to the evolutionary models of Baraffe et al. (2015) at four ages (10, 20, 30 and $40 \mathrm{Myr})$ 
Table 4. Summary of the properties of both TYC 9486-927-1 and 2MASS J2126-8140.

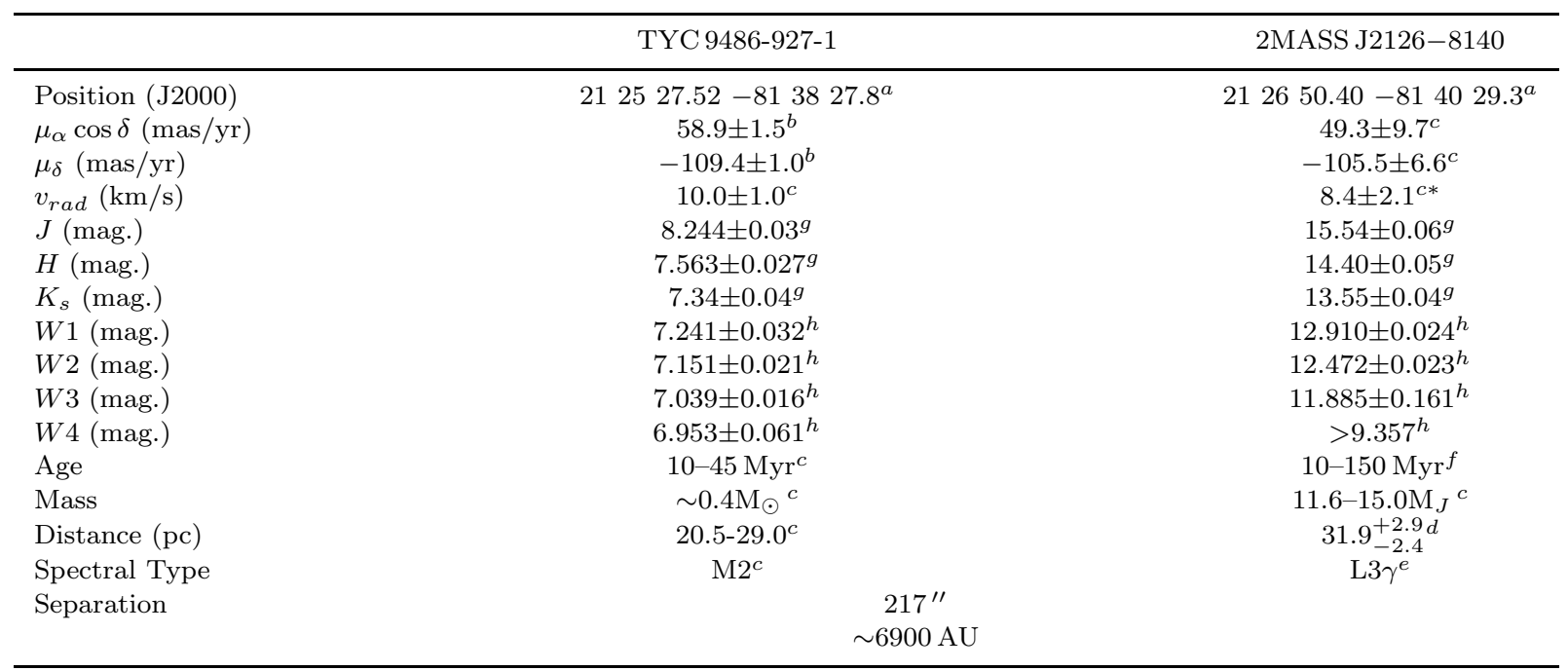

a 2MASS position, epoch 2000.644 Skrutskie et al. (2006)

${ }^{b}$ Zacharias et al. (2013)

$c$ This work

$d$ Trigonometric parallax mentioned in Filippazzo et al. (2015) citing Faherty et al. in prep.

$e$ Faherty et al. (2013)

${ }^{f}$ Filippazzo et al. (2015)

${ }^{g}$ Skrutskie et al. (2006)

$h$ Wright et al. (2010)

* Measurement from a very low signal to noise spectrum.

to estimate the absolute magnitudes. TYC 9486-927-1's $J, H$ and $K_{s}$ 2MASS photometry were then compared to the these absolute magnitudes to calculate distances, neglecting the likely negligible extinction. We took the mean of these estimates as the adopted distance for TYC 9486-927-1 for each age (see Table 5). Binarity would change the photometric distances although our multi-epoch RV measurements and the high resolution imaging of Elliott et al. (2015) show no evidence of a close companion to TYC 9486-927-1.

To compare to the trigonometric parallax quoted in Filippazzo et al. (2015) for 2MASS J2126-8140 we used the young L dwarf photometric distance relations of Gagné et al. (2015a). Following a similar process to that described above but adopting the scatter on the relations as the error on our distances. As the Gagné et al. (2015a) relations cover a wide range of ages (up to $125 \mathrm{Myr}$ ) they also cover a wide range of luminosities for each spectral type due to young objects having inflated radii. Hence the photometric distances do not deviate randomly across bands but will be correlated. Thus we do not adopt a weighted mean distance but take the distance from the band with the lowest scatter $\left(W 2, \mathrm{~d}=26.7_{-4.7}^{+5.7} \mathrm{pc}\right)$. This distance, and those for TYC 9486-927-1 using the 10 and 20 Myr Baraffe et al. (2015) models agree well with the trigonometric parallax presented by Filippazzo et al. (2015). 
Table 5. Photometric distances and moving group membership probabilities (TH - Tuc Hor, BP $\beta$ Pic, YF young field) for TYC 9486-927-1 and 2MASS J2126-8140. We assume errors of $\sim 20 \%$ for the photometric distance estimates for TYC 9486-927-1. Membership probabilities come from the BANYAN II online tool (Malo et al. 2013; Gagné et al. 2014) and use the proper motions from Zacharias et al. (2013) for TYC 9486-927-1 and our own calculation for 2MASS J2126-8140. Calculations using measured radial velocities for TYC 9486-927-1 $(10.0 \pm 1.0 \mathrm{~km} / \mathrm{s})$ and 2MASS J2126-8140 $(8.4 \pm 2.1 \mathrm{~km} / \mathrm{s})$ are marked accordingly. We note again this latter radial velocity is derived from a low signal to noise spectrum. See Table 4 for a full list of derived parameters. The final line for each object uses the trigonometric parallax mentioned in Filippazzo et al. (2015).

\begin{tabular}{|c|c|c|c|c|c|c|c|c|c|c|c|c|}
\hline Object & $\mathrm{SpT}$ & Age & $\mathrm{RV}(\mathrm{km} / \mathrm{s})$ & $d_{J}$ & $d_{H}$ & $d_{K}$ & $d_{W 1}$ & $d_{W 2}$ & $d_{\text {adopted }}$ & $p_{T H}$ & $p_{B P}$ & $p_{Y F}$ \\
\hline \multirow[t]{5}{*}{ TYC 9486-927-1 } & M2 & $10 \mathrm{Myr}$ & $10.0 \pm 1.0$ & $29.1 \pm 5.8$ & $28.9 \pm 5.8$ & $29.0 \pm 5.8$ & & & $29.0 \pm 5.8$ & $0.0 \%$ & $59.1 \%$ & $40.2 \%$ \\
\hline & M2 & $20 \mathrm{Myr}$ & $10.0 \pm 1.0$ & $26.4 \pm 5.3$ & $26.0 \pm 5.2$ & $26.1 \pm 5.2$ & & $\ldots$ & $26.2 \pm 5.2$ & $0.0 \%$ & $74.0 \%$ & $25.8 \%$ \\
\hline & M2 & $30 \mathrm{Myr}$ & $10.0 \pm 1.0$ & $22.8 \pm 4.6$ & $22.3 \pm 4.5$ & $22.5 \pm 4.5$ & & $\ldots$ & $22.6 \pm 4.5$ & $0.0 \%$ & $71.8 \%$ & $28.18 \%$ \\
\hline & M2 & $40 \mathrm{Myr}$ & $10.0 \pm 1.0$ & $20.7 \pm 4.1$ & $20.3 \pm 4.1$ & $20.5 \pm 4.1$ & & $\ldots$ & $20.5 \pm 4.1$ & $0.0 \%$ & $55.6 \%$ & $44.4 \%$ \\
\hline & $\cdots$ & $\ldots$ & $10.0 \pm 1.0$ & $\ldots$ & & $\ldots$ & & & $31.9_{-2.4}^{+2.9}$ & $1.6 \%$ & $9.5 \%$ & $88.3 \%$ \\
\hline \multirow[t]{4}{*}{ 2MASS J2126-8140 } & L3 & $<125 \mathrm{Myr}$ & $\ldots$ & $26.9_{-11.1}^{+19.0}$ & $26.2_{-7.4}^{+10.2}$ & $24.9_{-5.7}^{+7.3}$ & $26.7_{6.2}^{+8.0}$ & $26.7_{-4.7}^{+5.7}$ & $26.7_{-4.7}^{+5.4}$ & $0.0 \%$ & $29.8 \%$ & $68.8 \%$ \\
\hline & $\cdots$ & $\ldots$ & $\cdots$ & $\ldots$ & & $\ldots$ & $\ldots$ & $\ldots$ & $31.9_{-2.4}^{+2.9}$ & $0.3 \%$ & $4.9 \%$ & $91.7 \%$ \\
\hline & L3 & $<125 \mathrm{Myr}$ & $8.4 \pm 2.1$ & $26.9_{-11.1}^{+19.0}$ & $26.2_{-7.4}^{+10.2}$ & $24.9_{-5.7}^{+7.3}$ & $26.7_{-6.2}^{+8.0}$ & $26.7_{-4.7}^{+5.7}$ & $26.7_{-4.7}^{+5.7}$ & $0.0 \%$ & $69.4 \%$ & $30.6 \%$ \\
\hline & & & $8.4 \pm 2.1$ & $\ldots$ & & $\ldots$ & & $\ldots$ & $31.9_{-2.4}^{+2.9}$ & $1.0 \%$ & $34.8 \%$ & $64.0 \%$ \\
\hline
\end{tabular}

To test the membership of TYC 9486-927-1 in several well known moving groups in the solar neighbourhood, we imputed our radial velocity $(10 \pm 1 \mathrm{~km} / \mathrm{s})$, the UCAC4 proper motions and the positions, along with the distance estimates for each age, into the BANYAN II young moving group membership probability estimation tool (Malo et al. 2013; Gagné et al. 2014). We assumed a $20 \%$ error on our photometric distance estimates and that the objects were younger than 1 Gyr. For 2MASS J2126-8140 we used our proper motion, and both the photometric distance estimate and the literature trigonometric parallax. The results of these calculations are shown in Table 5. They suggest that the system is unlikely to be a TucHor member. Over the range of estimated photometric distances in Table 5 , BANYAN II provides probabilities of $\beta$ Pic membership ranging from about 4.9 to $74 \%$. The membership probability for 2MASS J2126-8140 is on the lower end of this range when we allow the radial velocity to be unconstrained. To further investigate the potential moving group membership we plotted the Galactic $U, V, W$ velocities and $X, Y, Z$ positions for TYC 9486927-1 and 2MASS J2126-8140 (Figure 5). We find that the reason BANYAN discounts TucHor membership is due to the system being a significant outlier in the $Z$ coordinate. While $\beta$ Pic remains the most likely moving group (both in kinematics and in age) for this system to be associated with, a difference in the $U$ velocity precludes us from claiming this is a bona-fide $\beta$ Pic member.

\subsection{Probability of chance alignment}

While it appears that TYC 9486-927-1 and 2MASS J2126-8140 have matching proper motions and distances and both show signs of youth it is possible that they are a chance 

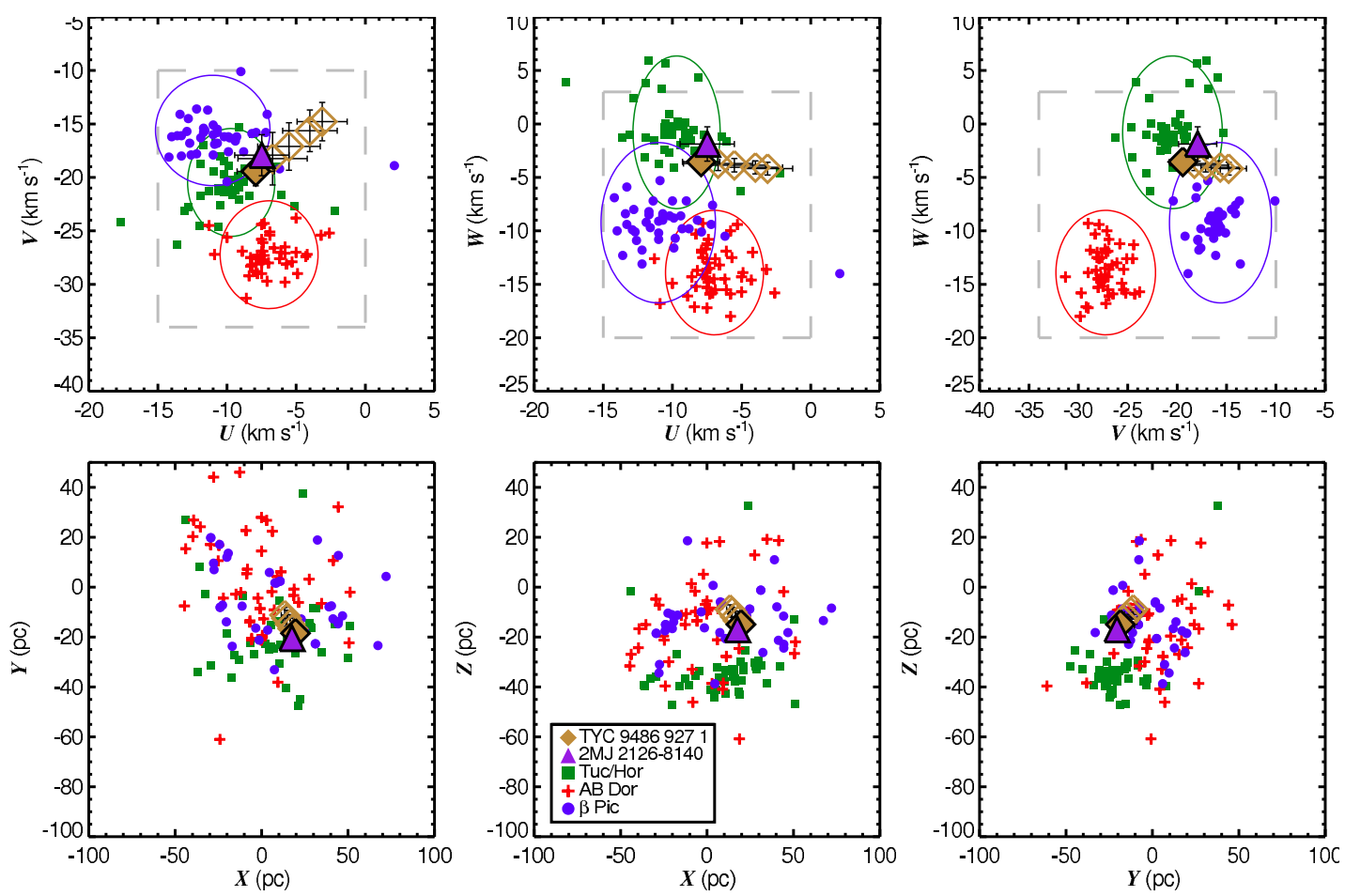

Figure 5. Projections of Galactic velocities and positions for members of the Tucana/Horologium association, the AB Doradus moving group, and the $\beta$ Pictoris moving group (Malo et al. 2013) along with $3 \sigma$ velocity ellipsoids (Gagné et al. 2014). Top panels: We show for comparison the UVW velocities of TYC9486 using the measured proper motions, RV, and a range photometric distances for estimated ages 10-40 Myr (open data points left to right) and the secondary trigonometric parallax from Filippazzo et al. (2015) (solid symbol). The velocities of 2MASS J2126-8140 are calculated using our proper motion, the RV from the low signal to noise Phoenix spectrum, and the parallax distance from Filippazzo et al. (2015). The velocities of both compoents are approximately consistent with both TucHor and $\beta$ Pic for distances around 30 pc. Bottom panels: Same as the top but for XYZ positions the positions of the primary have been offset by $+2 \mathrm{pc}$ for clarity. The $10-40$ Myr photometric distance estimates for TYC9486 (bottom to top) and the trigonometric distance for $2 \mathrm{MJ} 2126$ are consistent with the XYZ distribution of $\beta$ Pic but are TucHor outliers in Z.

alignment of unrelated young objects. To determine the likelihood of this, we modified the method of Lépine \& Bongiorno (2007). We first constructed a list of known and candidate young stars in nearby young moving groups from Torres et al. (2008), Shkolnik et al. (2009), Schlieder et al. (2012), Kraus et al. (2014) and Malo et al. (2014). We then offset the positions of these stars by two degrees and searched for common proper motion companions in the 2MASS-WISE proper motion catalogue of Gagné et al. (2015b) around these offset positions. This should result in only chance alignments of unrelated objects. We ran this process 18 times, on each occasion offsetting the positions of our input sample by 2 degrees but changing the direction of the offset by one-ninth of a radian each time. In this way we were able to sample a much larger area for chance alignments and thus reduce statistical noise. The results are shown in Figure 6 and clearly show a low probability $(\sim 5 \%)$ of chance alignment considering all objects in Gagne's proper motion sample or only those with 
L dwarf-like colours $\left(J-K_{s}>1.2\right)$. There is also the possibility of the chance alignment between two unbound objects in the same moving group. Hence we carried out a simulation to see how often two members of one group would fall close to each other on the sky and have photometric distance estimates within $10 \mathrm{pc}$ of each other. To accomplish this we generated a random realisation of each of the eight young moving groups described in Gagné et al. (2014) using the parameters provided by that work. We then ran this simulation 50,000 times and determined that there is $<1 \%$ chance that two objects in the same moving group would lie as close together on the sky as TYC 9486-927-1 and 2MASS J2126-8140 (see Figure 6 lowest panel). We assumed that all chance alignments between members of one moving group would have matching proper motions due to both components having the bulk space velocity of the moving group. Note we did not consider the number of pairings between members of different moving groups (i.e. the number of chance alignments between AB Dor and TWA members). Even accounting for a factor of 2 or 3 missing members in the groups (especially at the lowest masses), it is clear that TYC 9486-927-1 and 2MASS J2126-8140 are unlikely to be chance alignments inside the same group.

\section{PHYSICAL PROPERTIES OF 2MASS J21265040-8140293}

In order to estimate the mass of 2MASS J2126-8140, we used a Monte Carlo simulation. We drew temperatures from a flat distribution from the 1700-1900 K range quoted by Manjavacas et al. (2014) and ages from our flat 10-45 Myr range. We then compared with the COND (Baraffe et al. 2003) and Saumon \& Marley $(2008)\left(f_{\text {sed }}=2\right)$ evolutionary models. The Baraffe et al. (2003) models yielded masses between $11.6 M_{\text {Jup }}$ and $14.7 M_{\text {Jup }}$ 5 and the Saumon \& Marley (2008) models preferred solutions in the $13.3-15 M_{J u p}$ range. Hence we adopt a mass range of 11.6-15 $M_{\text {Jup }}$ range for 2MASS J2126-8140 placing it on the $13 M_{\text {Jup }}$ deuterium-burning dividing line between planets and brown dwarfs. A similar calculation drawing the age from a (Bell et al. 2015) $\beta$ Pic Gaussian age distribution of $24 \pm 3$ Myr yields a mass range of $12-14 M_{J}$. Such masses and ages make 2MASS J2126-8140

an interesting wide-orbit analogue to $\beta$ Pic b (Lagrange et al. 2010), whose primary is a member of the eponymous moving group.. Morzinski et al. (2015) find that $\beta$ Pic b has a mass of $12.7 \pm 0.3 M_{J u p}$ and $T_{\text {eff }}$ of $1708 \pm 23 \mathrm{~K}$ whilst Bonnefoy et al. (2013) find a spectral type of $\mathrm{L} 1_{-1.5}^{+1.0}, T_{\text {eff }}=1700 \pm 100 \mathrm{~K}, \log g=4.0 \pm 0.5 \mathrm{dex}$ and mass of $10_{-2}^{+3} M_{\text {Jup }}$ from $T_{\text {eff }}$

\footnotetext{
5 The full range of masses derived, not a confidence interval.
} 

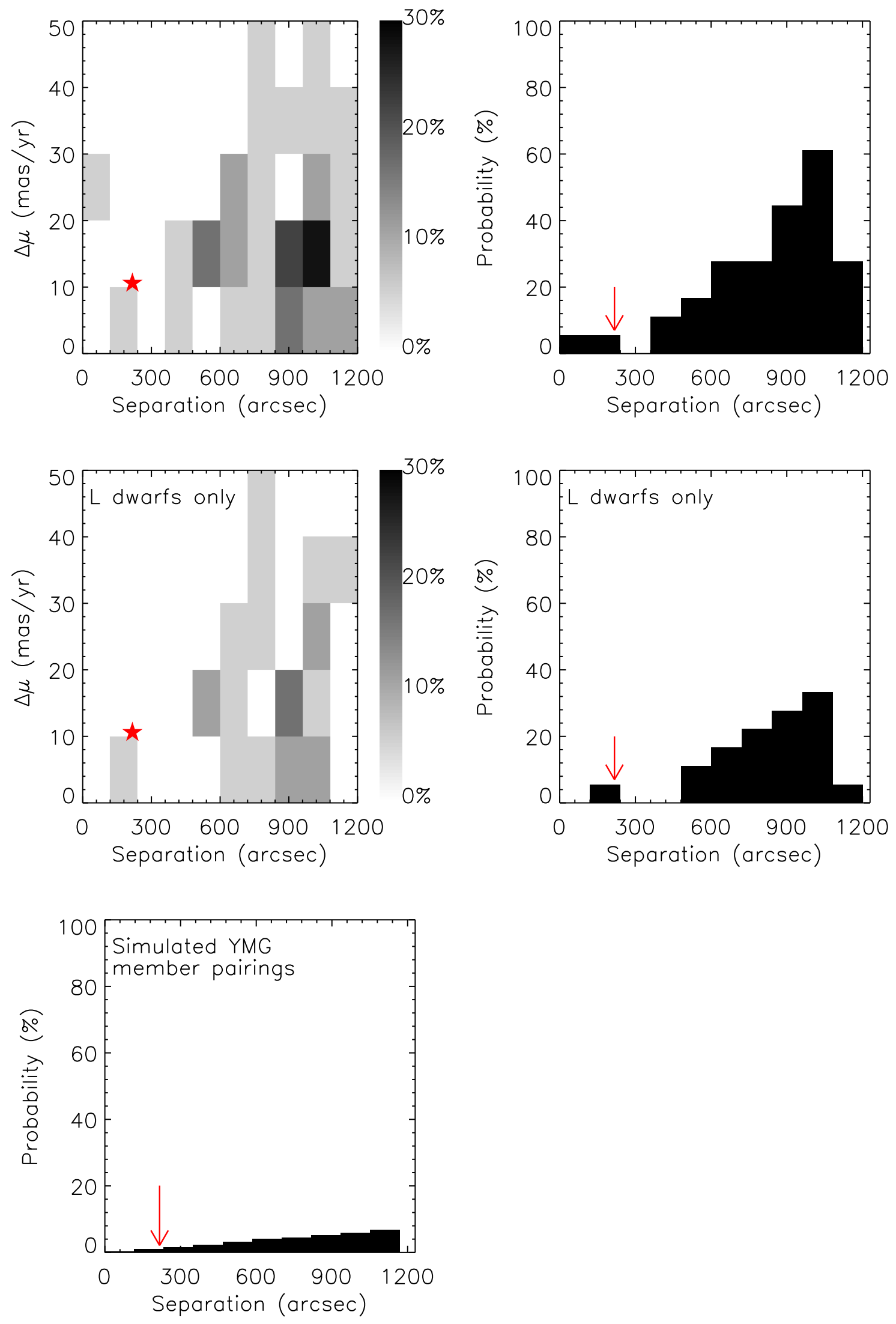

Figure 6. Top two rows:The results of our chance alignment using a modification of the offset position method of Lépine \& Bongiorno (2007). The top row includes matches with all objects in Gagné et al. (2015b)'s catalogue while the middle row only includes matches with objects with $J-K_{s}>1.2$. Bottom row: The probability of finding two members of the same moving group at a particular separation on the sky from each other and with distances which agree within 10 pc. 
and $9_{-2}^{+3} M_{J u p}$ from luminosity. These compare well with our derived age range, $11.6-15 M_{J u p}$ mass range and Manjavacas et al. (2014)'s $T_{\text {eff }}=1800 \pm 100 \mathrm{~K}$ and $\log g=4.0 \pm 0.5$ dex for 2MASS J2126-8140. We note that $\beta$ Pic b is commonly referred to as a planet and also has mass estimates straddling the deuterium burning limit. Our system is also a younger analogue of the AB Dor (149-19 Myr; Bell et al. 2015) member GU Psc A/b system (Naud et al. 2014) with both components having similar masses.

The range of photometric distances for the system gives projected separations of 4450$5700 \mathrm{AU}$ and the trigonometric parallax of the secondary a separation of $6900 \dot{\mathrm{A}} \mathrm{U}$, wider than any star-planet system in the exoplanet.eu database (http://exoplanet.eu/).

\section{CONCLUSIONS}

In summary: we have identified two previously known young objects TYC 9486-927-1 and 2MASS J2126-8140 as having common proper motion. We find that the photometric distances of the pair agree and that they are unlikely to be an alignment of two unrelated young objects. Using the strength of the lithium $6708 \AA$ feature we find an age range of 10-45 Myr for TYC 9486-927-1 yielding a mass of 11.6-15M $M_{J}$ for 2MASS J2126-8140. We note that the system has a wider separation than any known star-planet system and that 2MASS J2126-8140 is similar in age, mass and temperature to the known exoplanet $\beta$ Pic b.

\section{ACKNOWLEDGMENTS}

This research made use of the SIMBAD database, operated at CDS, Strasbourg, France. This publication makes use of data products from the Two Micron All Sky Survey, which is a joint project of the University of Massachusetts and the Infrared Processing and Analysis Center/California Institute of Technology, funded by the National Aeronautics and Space Administration and the National Science Foundation. This publication also makes use of data products from NEOWISE, which is a project of the Jet Propulsion Laboratory/California Institute of Technology, funded by the Planetary Science Division of the National Aeronautics and Space Administration. The DENIS project has been partly funded by the SCIENCE and the HCM plans of the European Commission under grants CT920791 and CT940627. It is supported by INSU, MEN and CNRS in France, by the State of Baden-Württemberg in Germany, by DGICYT in Spain, by CNR in Italy, by FFwFBWF in Austria, by FAPESP in 
Brazil, by OTKA grants F-4239 and F-013990 in Hungary, and by the ESO C\&EE grant A04-046. Jean Claude Renault from IAP was the Project manager. Observations were carried out thanks to the contribution of numerous students and young scientists from all involved institutes, under the supervision of P. Fouqué, survey astronomer resident in Chile. Based on observations obtained at the Gemini Observatory (PID GS-2009B-C-2, acquired through the Gemini Science Archive), which is operated by the Association of Universities for Research in Astronomy, Inc., under a cooperative agreement with the NSF on behalf of the Gemini partnership: the National Science Foundation (United States), the National Research Council (Canada), CONICYT (Chile), the Australian Research Council (Australia), Ministério da Ciência, Tecnologia e Inovação (Brazil) and Ministerio de Ciencia, Tecnología e Innovación Productiva (Argentina). The research of J.E.S. was supported by an appointment to the NASA Postdoctoral Program at NASA Ames Research Center, administered by Oak Ridge Associated Universities through a contract with NASA. We thank the anonymous referee for the prompt and helpful review that improved the quality and clarity of this manuscript. The authors would like to the thank the Brass Monkey, Heidelberg for the opportunity to pit their wits against each other every week.

\section{REFERENCES}

Allard F., Homeier D., Freytag B., 2011, in 16th Cambridge Work. Cool Stars. Stellar Syst. Sun, Johns-Krull C. M., Browning M. K., West A. A., eds., ASP Conference Series Vol. 448, San Francisco, p. 91

Allard F., Homeier D., Freytag B., 2012, Philos. Trans. R. Soc. A Math. Phys. Eng. Sci., 370,2765

Allers K. N., Liu M. C., 2013, Astrophys. J., 772, 79

Baraffe I., Chabrier G., Barman T. S., Allard F., Hauschildt P. H., 2003, Astron. Astrophys., 712,701

Baraffe I., Homeier D., Allard F., Chabrier G., 2015, Astron. Astrophys., 577, A42

Bell C. P. M., Mamajek E. E., Naylor T., 2015, Mon. Not. R. Astron. Soc., 454, 593

Bonnefoy M. et al., 2013, Astron. Astrophys., 555, A107

Bowler B. P. et al., 2015, Astrophys. J., 806, 62

Cruz K. L., Kirkpatrick J. D., Burgasser A. J., 2009, Astron. J., 137, 3345 
da Silva L., Torres C. A. O., de la Reza R., Quast G. R., Melo C. H. F., Sterzik M. F., 2009, Astron. Astrophys., 508, 833

Dopita M., Hart J., McGregor P., Oates P., Bloxham G., Jones D., 2007, Astrophys. Space Sci., 310,255

Elliott P. et al., 2015, Astron. Astrophys., 580, A88

Epchtein N. et al., 1994, Astrophys. Space Sci., 217, 3

Faherty J. K., Rice E. L., Cruz K. L., Mamajek E. E., Núñez A., 2013, Astron. J., 145, 2

Filippazzo J. C., Rice E. L., Faherty J., Cruz K. L., Van Gordon M. M., Looper D. L., 2015, Astrophys. J., 810, 158

Gagné J. et al., 2015a, Astrophys. J. Suppl. Ser., 219, 33

Gagné J., Lafrenière D., Doyon R., Malo L., Artigau É., 2014, Astrophys. J., 783, 121

Gagné J., Lafrenière D., Doyon R., Malo L., Artigau É., 2015b, Astrophys. J., 798, 73

Gaidos E. et al., 2014, Mon. Not. R. Astron. Soc., 443, 2561

Goldman B., Marsat S., Henning T., Clemens C., Greiner J., 2010, Mon. Not. R. Astron. Soc., 405, 1140

Hinkle K. H. et al., 2003, in SPIE 4834, Discov. Res. Prospect. from 6- to 10-Meter-Class

Telesc. II, Guhathakurta P., ed., pp. 353-363

Kaufer A., Stahl O., Tubbesing S., N $\{\{\backslash 0\}\}$ rregaard P., Avila G., Francois P., Pasquini L., Pizzella A., 1999, The Messenger, 95, 8

Kraus A. L., Shkolnik E. L., Allers K. N., Liu M. C., 2014, Astron. J., 147, 146

Kuzuhara M., Tamura M., Ishii M., Kudo T., Nishiyama S., Kandori R., 2011, Astron. J., 141,119

Lagrange A.-M. et al., 2010, Science, 329, 57

Lépine S., Bongiorno B., 2007, Astron. J., 133, 889

Lépine S., Hilton E. J., Mann A. W., Wilde M., Rojas-Ayala B., Cruz K. L., Gaidos E., 2013, Astron. J., 145, 102

Luhman K. L., 2013, Astrophys. J., 767, L1

Luhman K. L., Burgasser A. J., Bochanski J. J., 2011, Astrophys. J., 730, L9

Luhman K. L. et al., 2012, Astrophys. J., 760, 152

Mainzer A. et al., 2011, Astrophys. J., 731, 53

Malo L., Artigau É., Doyon R., Lafrenière D., Albert L., Gagné J., 2014, Astrophys. J., 788,81

Malo L., Doyon R., Lafrenière D., Artigau É., Gagné J., Baron F., Riedel A., 2013, Astro- 
phys. J., 762, 88

Manjavacas E. et al., 2014, Astron. Astrophys., 564, A55

Martin D. C. et al., 2005, Astrophys. J., 619, L1

Mentuch E., Brandeker A., van Kerkwijk M. H., Jayawardhana R., Hauschildt P. H., 2008, Astrophys. J., 689, 1127

Morzinski K. M. et al., 2015

Murphy S. J., Lawson W. A., 2014, Mon. Not. R. Astron. Soc., 447, 1267

Naud M.-E. et al., 2014, Astrophys. J., 787, 5

Pecaut M. J., Mamajek E. E., 2013, Astrophys. J. Suppl. Ser., 208, 9

Prato L., Simon M., Mazeh T., McLean I. S., Norman D., Zucker S., 2002, Astrophys. J., 569,863

Reid I., Cruz K. L., Kirkpatrick J. D., Allen P. R., Mungall F., Liebert J., Lowrance P., Sweet A., 2008, Astron. J., 136, 1290

Saumon D., Marley M. S., 2008, Astrophys. J., 689, 1327

Schlieder J. E., Lépine S., Simon M., 2012, Astron. J., 143, 80

Shkolnik E., Liu M. C., Reid I. N., 2009, Astrophys. J., 699, 649

Skrutskie M. F. et al., 2006, Astron. J., 131, 1163

Thomas H.-C., Beuermann K., Reinsch K., Schwope A. D., Truemper J., Voges W., 1998, Astron. Astrophys., 335, 467

Torres C. A. O., Quast G. R., da Silva L., de la Reza R., Melo C. H. F., Sterzik M., 2006, Astron. Astrophys., 460, 695

Torres C. A. O., Quast G. R., Melo C. H. F., Sterzik M. F., 2008, in Handb. Star Form. Reg. Vol. II South. Sky, Reipurth B., ed., ASP Monograph Publications, Vol. 5., p. 757 Wahhaj Z. et al., 2011, Astrophys. J., 729, 139

Wright E. L. et al., 2010, Astron. J., 140, 1868

Zacharias N., Finch C. T., Girard T. M., Henden A., Bartlett J. L., Monet D. G., Zacharias M. I., 2013, Astron. J., 145, 44 